Supporting Information

\title{
Temperature-Directed Assembly of Crystalline Cellulose Oligomers into Kinetically Trapped Structures during Biocatalytic Synthesis
}

Yuuki Hata, ${ }^{\dagger}$ Toshiki Sawada, ${ }^{\dagger}+{ }^{\dagger}$ Hironori Marubayashi, ${ }^{\dagger}{ }^{\dagger}$ Shuichi Nojima, ${ }^{\dagger}$ and Takeshi Serizawa*,

${ }^{\dagger}$ Department of Chemical Science and Engineering, School of Materials and Chemical Technology, Tokyo Institute of Technology, 2-12-1 Ookayama, Meguro-ku, Tokyo 152-8550, Japan. E-mail: serizawa@polymer.titech.ac.jp

¥ Precursory Research for Embryonic Science and Technology (PRESTO), Japan Science and Technology Agency (JST), 4-1-8 Honcho, Kawaguchi-shi, Saitama 332-0012, Japan

$\S$ Present address: Institute of Multidisciplinary Research for Advanced Materials, Tohoku University, 2-1-1 Katahira, Aoba-ku, Sendai 980-8577, Japan 
Figure S1. MALDI-TOF mass spectra of the products prepared at various temperatures. S3

Figure S2. ATR-FTIR absorption spectra of the products prepared at various temperatures. S3

Figure S3. $X_{\mathrm{c}}$ versus PDI of the products prepared at various temperatures. $\quad$ S4

Figure S4. Schematic illustration of cellulose oligomers with different lengths forming an antiparallel cellulose II crystal together.

Figure S5. Schematic illustration of a lamellar cellulose II crystal composed of cellulose

oligomers.

Figure S6. Low-magnification SEM image of the gels prepared at $30{ }^{\circ} \mathrm{C} . \quad \mathrm{S} 5$

Figure S7. The inversion test for the reaction mixture after incubation at $20{ }^{\circ} \mathrm{C}$ for $168 \mathrm{~h} . \quad \mathrm{S} 5$

Figure S8. Time-course of $\alpha \mathrm{G} 1 \mathrm{P}$ monomer conversion at various temperatures. S6

Figure S9. Photographs of dispersions of the as-prepared nanosheets $(0.02 \%(\mathrm{w} / \mathrm{v})) . \quad$ S7

Figure S10. Photographs of the reaction mixtures with particles after incubation at $60{ }^{\circ} \mathrm{C}$. S7

Figure S11. ${ }^{1} \mathrm{H}$ NMR spectra of the products prepared at $30{ }^{\circ} \mathrm{C}$ with particles.

Figure S12. MALDI-TOF mass spectra of the products prepared at $30^{\circ} \mathrm{C}$ with particles. $\quad \mathrm{S} 8$

Figure S13. XRD profiles of the products prepared at $30{ }^{\circ} \mathrm{C}$ with particles. S8

Figure S14. ATR-FTIR absorption spectra of the products prepared at $30{ }^{\circ} \mathrm{C}$ with particles. S9

Figure S15. AFM images of the products prepared at $30^{\circ} \mathrm{C}$ with particles. S9 


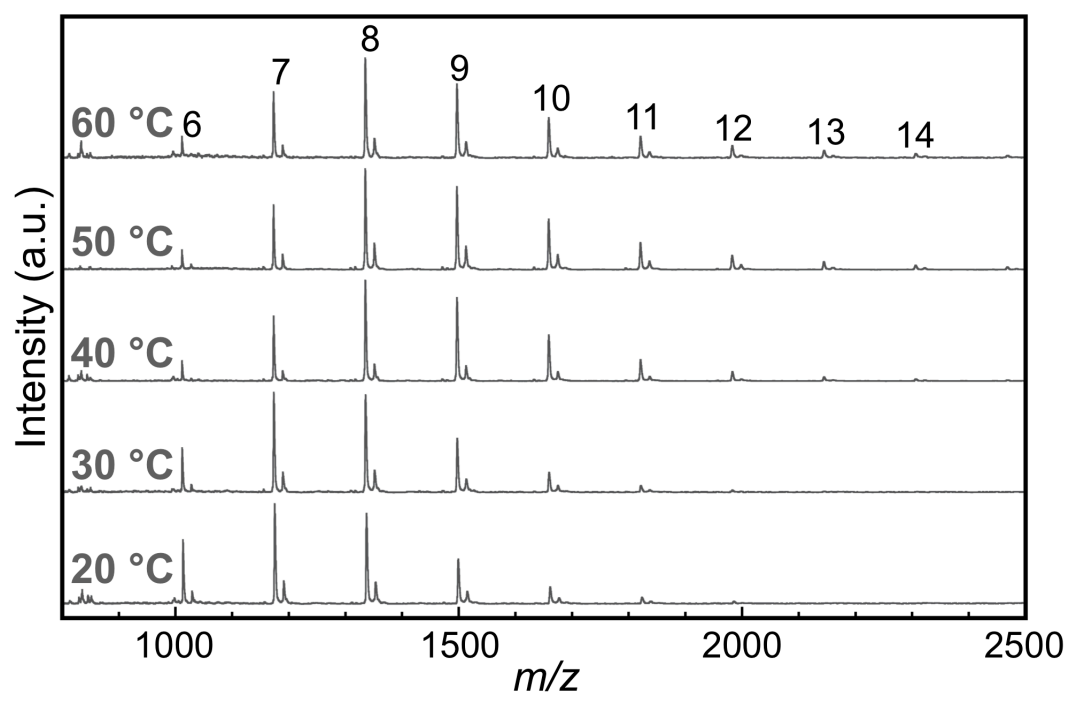

Figure S1. Matrix-assisted laser desorption/ionization time-of-flight (MALDI-TOF) mass spectra of the products prepared at various temperatures. The numbers above the peaks denote the DP values of the cellulose oligomers. The spectra show two series of peaks corresponding to cellulose oligomers with sodium and potassium ion adducts.

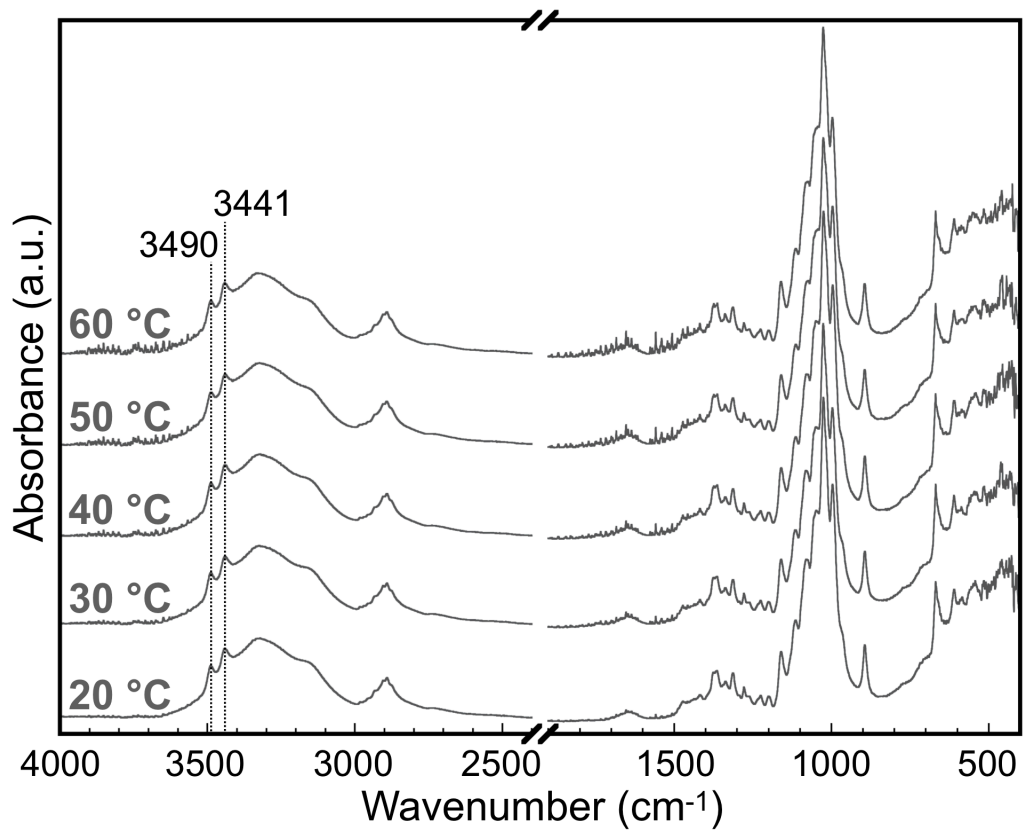

Figure S2. Attenuated total reflection-Fourier transform infrared (ATR-FTIR) absorption spectra of the products prepared at various temperatures. The numbers above the peaks denote the wavenumber. 


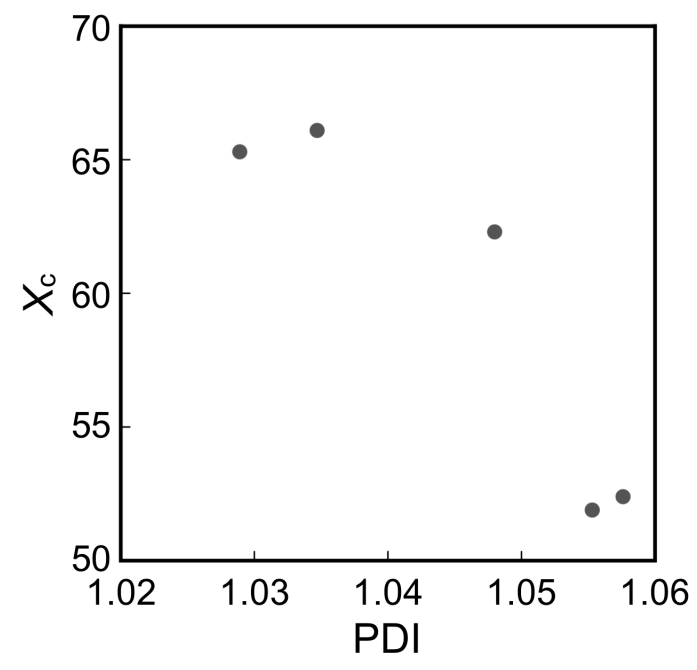

Figure S3. The degree of crystallinity $\left(X_{\mathrm{c}}\right)$ versus the polydispersity index (PDI) of the products prepared at various temperatures.

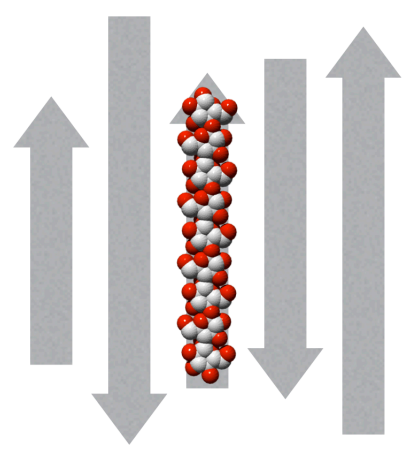

Figure S4. Schematic illustration of cellulose oligomers with different lengths forming an antiparallel cellulose II crystal together. The termini of longer chains neighboring shorter chains are plausibly the main amorphous regions.

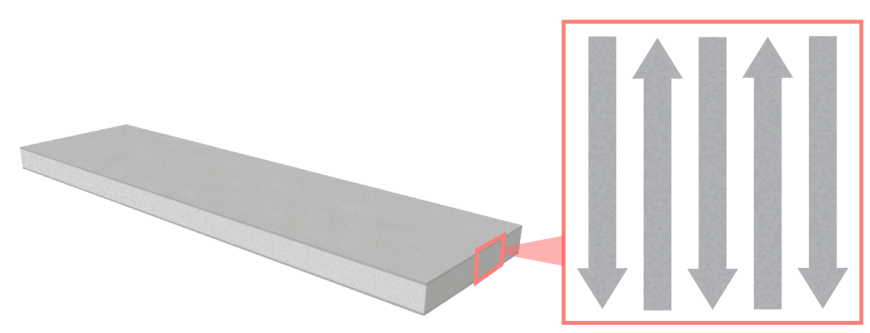

Figure S5. Schematic illustration of a lamellar cellulose II crystal composed of cellulose oligomers. Note that the polydispersity in the chain length of cellulose oligomers was not taken into account. 


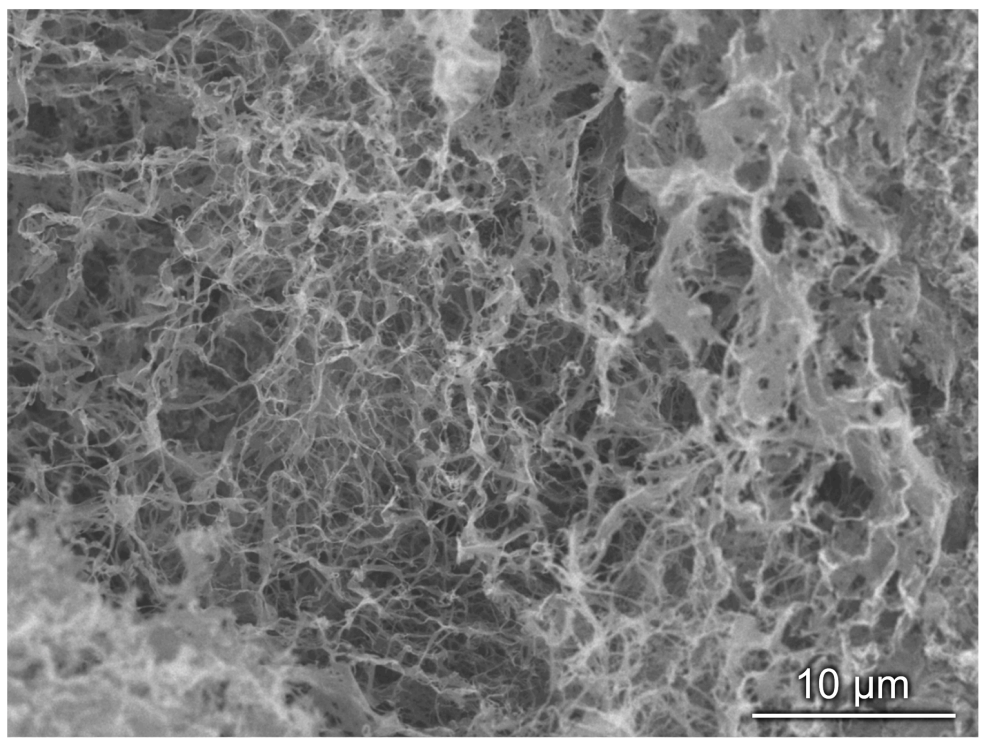

Figure S6. Low-magnification scanning electron microscopy (SEM) image of the xerogels prepared from the hydrogels synthesized at $30^{\circ} \mathrm{C}$.

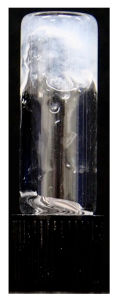

Figure S7. The inversion test for the reaction mixture after incubation at $20{ }^{\circ} \mathrm{C}$ for $168 \mathrm{~h}$. 
(a) $60^{\circ} \mathrm{C}$

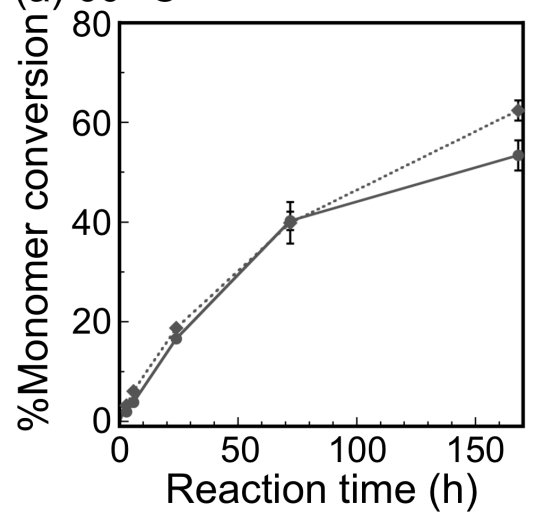

(c) $40{ }^{\circ} \mathrm{C}$

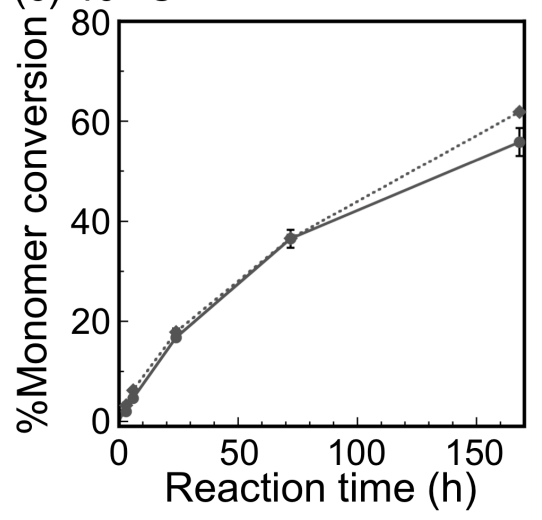

(e) $20{ }^{\circ} \mathrm{C}$

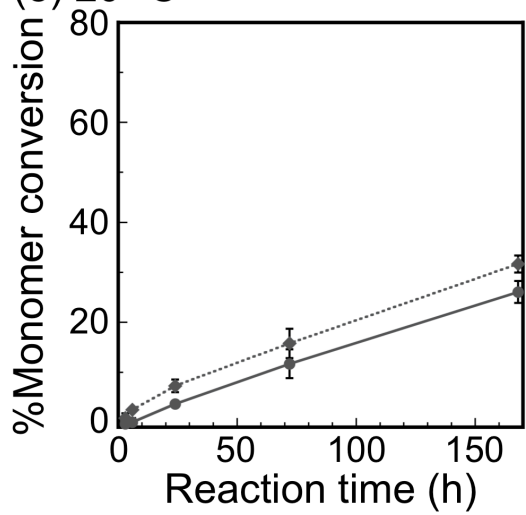

(b) $50{ }^{\circ} \mathrm{C}$

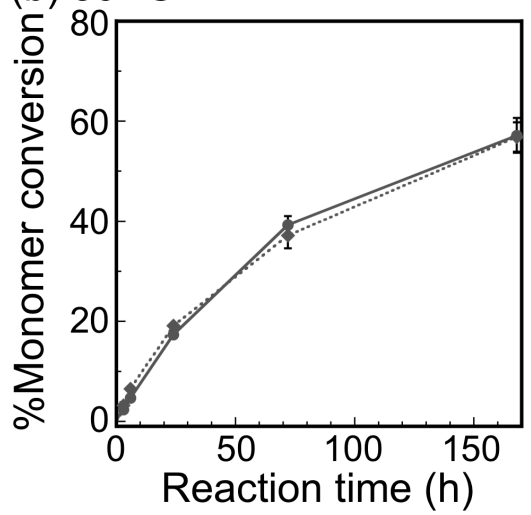

(d) $30{ }^{\circ} \mathrm{C}$

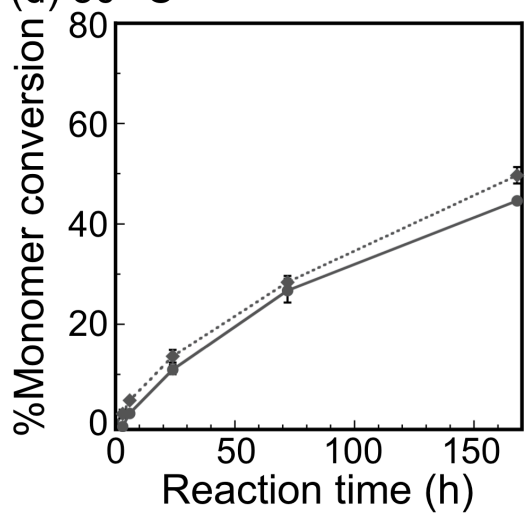

Figure S8. Time-course of $\alpha$ G1P monomer conversion at (a) 60, (b) 50, (c) 40, (d) 30, and (e) $20{ }^{\circ} \mathrm{C}$. Circles with solid lines and squares with dotted lines show the monomer conversion into insoluble cellulose oligomers and the total monomer conversion, respectively. 
(a)

(b)
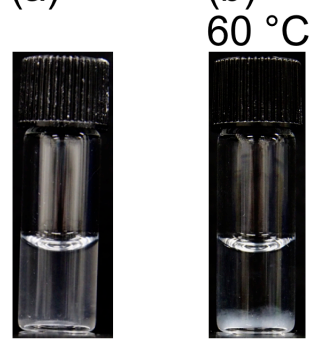

(c)

(d)

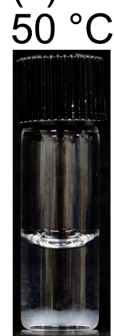

$40^{\circ}$

(e)

$30^{\circ} \mathrm{C}$

(f)

$20^{\circ}$
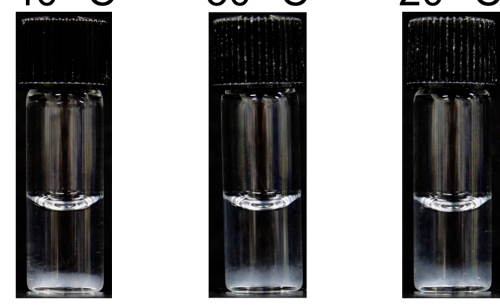

Figure S9. Photographs of dispersions of the as-prepared nanosheets $(0.02 \%(w / v))$ (a) before and after incubation for $1 \mathrm{~h}$ at (b) 60, (c) 50, (d) 40, (e) 30, and (f) $20^{\circ} \mathrm{C}$.

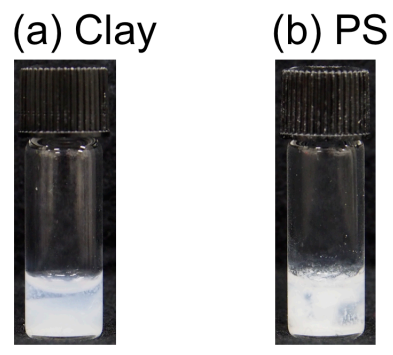

Figure S10. Photographs of the reaction mixtures with (a) clay and (b) PS particles after incubation at $60{ }^{\circ} \mathrm{C}$ for $3 \mathrm{~d}$.
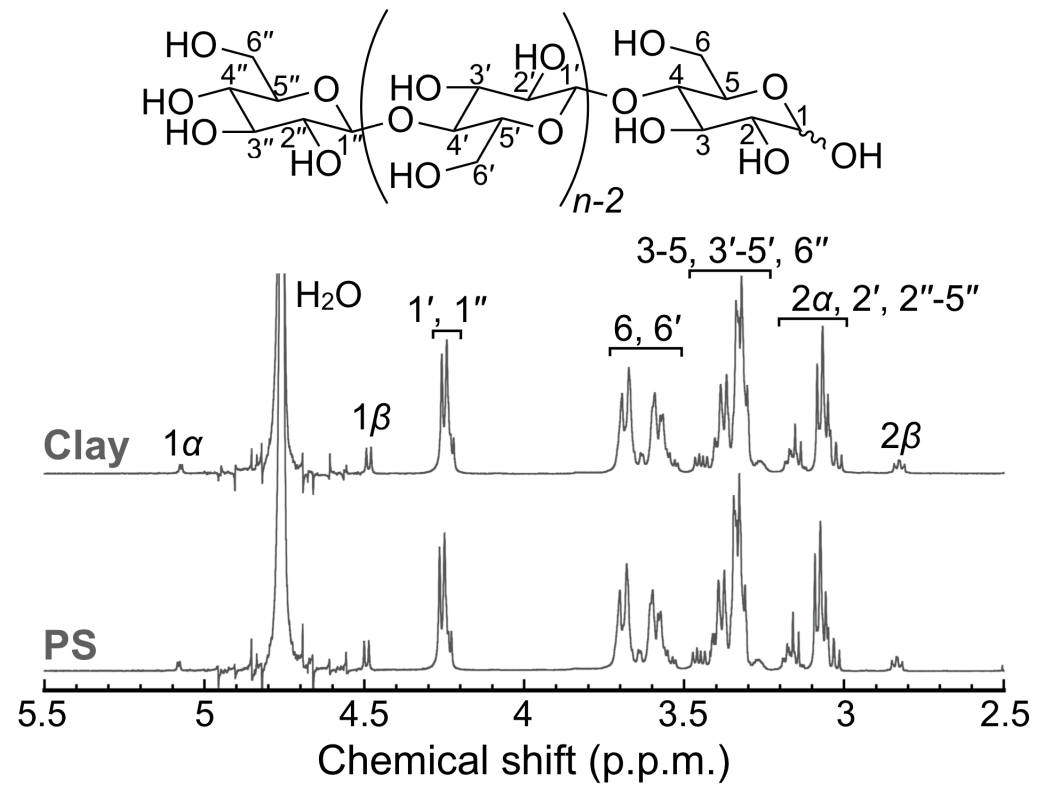

Figure S11. ${ }^{1} \mathrm{H}$ nuclear magnetic resonance (NMR) spectra of the products prepared at $30{ }^{\circ} \mathrm{C}$ with particles. 


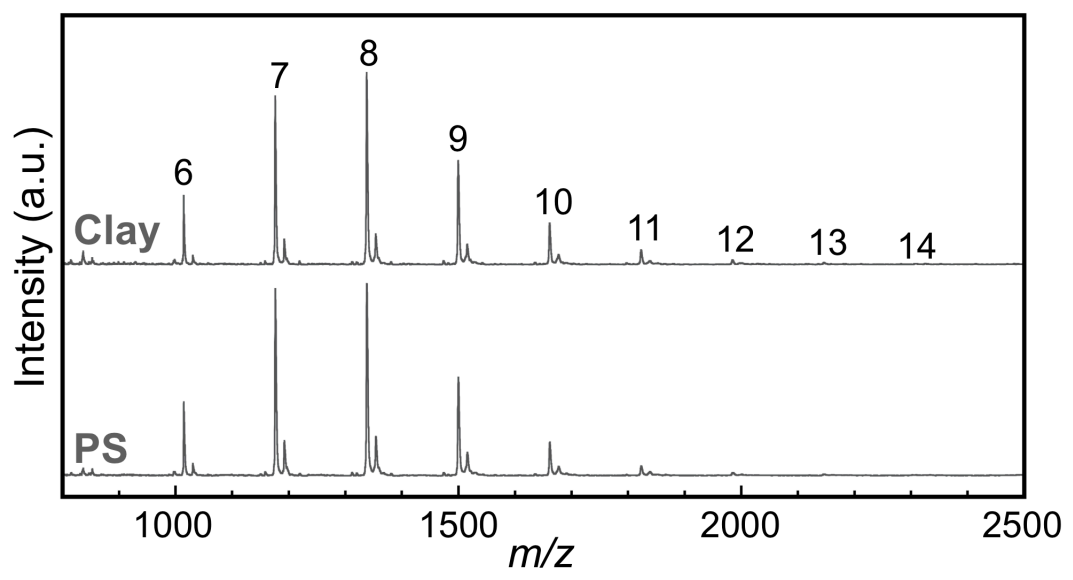

Figure S12. MALDI-TOF mass spectra of the products prepared at $30{ }^{\circ} \mathrm{C}$ with particles. The numbers above the peaks denote the DP values of the cellulose oligomers. The spectra show two series of peaks corresponding to cellulose oligomers with sodium and potassium ion adducts.

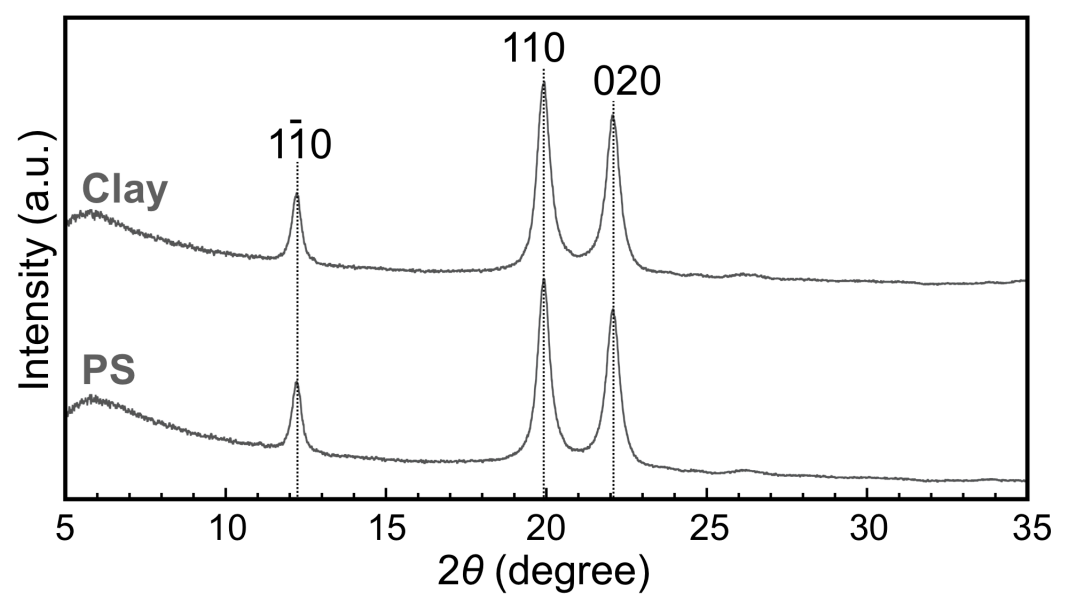

Figure S13. X-ray diffraction (XRD) profiles of the products prepared at $30{ }^{\circ} \mathrm{C}$ with particles. 


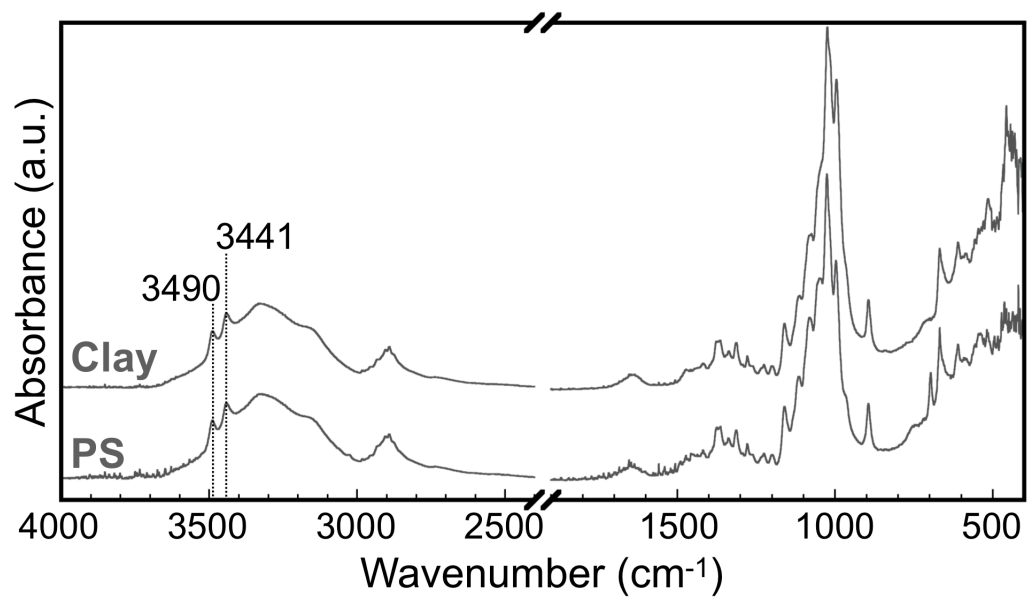

Figure S14. ATR-FTIR absorption spectra of the products prepared at $30{ }^{\circ} \mathrm{C}$ with particles. The numbers above the peaks denote the wavenumber.

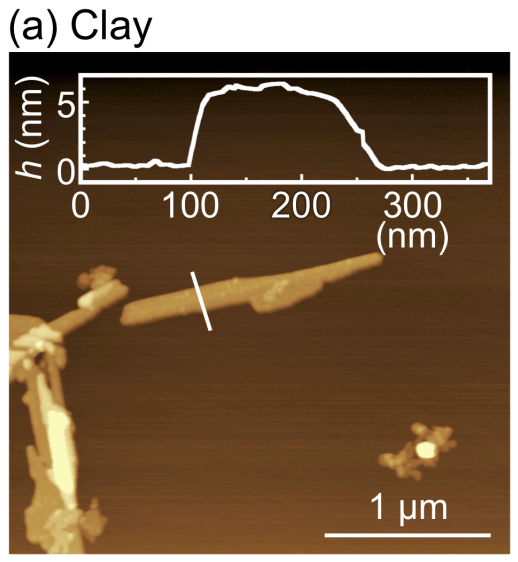

(b) PS

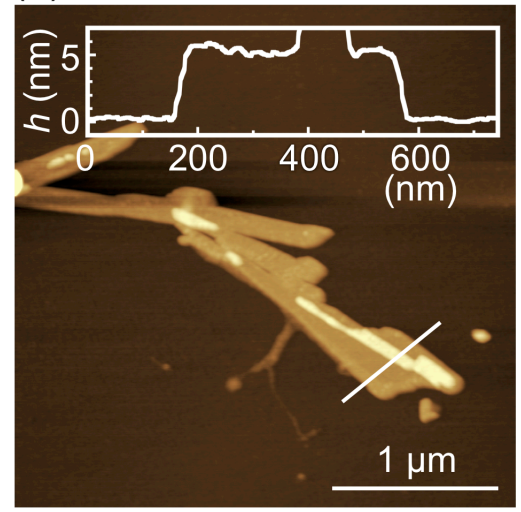

Figure S15. Atomic force microscopy (AFM) images of the products prepared at $30{ }^{\circ} \mathrm{C}$ with (a) clay and (b) PS particles. 